\title{
Hamiltonian Dynamics of Preferential Attachment
}

\author{
Konstantin Zuev, ${ }^{1}$ Fragkiskos Papadopoulos, ${ }^{2}$ and Dmitri Krioukov ${ }^{3}$ \\ ${ }^{1}$ Department of Physics, Northeastern University, Boston, MA 02115, USA \\ ${ }^{2}$ Department of Electrical Engineering, Computer Engineering and Informatics, \\ Cyprus University of Technology, 33 Saripolou Street, 3036 Limassol, Cyprus \\ ${ }^{3}$ Department of Physics, Department of Mathematics, \\ Department of Electrical\&Computer Engineering, \\ Northeastern University, Boston, MA 02115, USA
}

\begin{abstract}
Prediction and control of network dynamics are grand-challenge problems in network science. The lack of understanding of fundamental laws driving the dynamics of networks is among the reasons why many practical problems of great significance remain unsolved for decades. Here we study the dynamics of networks evolving according to preferential attachment, known to approximate well the large-scale growth dynamics of a variety of real networks. We show that this dynamics is Hamiltonian, thus casting the study of complex networks dynamics to the powerful canonical formalism, in which the time evolution of a dynamical system is described by Hamilton's equations. We derive the explicit form of the Hamiltonian that governs network growth in preferential attachment. This Hamiltonian turns out to be nearly identical to graph energy in the configuration model, which shows that the ensemble of random graphs generated by preferential attachment is nearly identical to the ensemble of random graphs with scale-free degree distributions. In other words, preferential attachment generates nothing but random graphs with power-law degree distribution. The extension of the developed canonical formalism for network analysis to richer geometric network models with non-degenerate groups of symmetries may eventually lead to a system of equations describing network dynamics at small scales.
\end{abstract}

PACS numbers: 89.75.Hc, 89.75.Fb, 45.20.Jj, 05.65.+b

\section{INTRODUCTION}

Large real networks-social, biological, or technological-are complex dynamical systems [13. Understanding the dynamics of these systems is a key to better prediction and control of their behavior, and the behavior of the processes running on them, such as epidemic spreading [4, 5] and cascading failure propagation 6, 7. Network dynamics can be roughly split into two categories: large-scale and small-scale. Large-scale dynamics usually means network growth, for example the growth of the Internet over years. Small-scale dynamics refers to the dynamics of links in a given network at small time scales, for example real-time interactions among mobile phone users or genes in a cell. It is quite unlikely that the small-scale dynamics of different networks can be in any way similar, so it seems that natural options to study and predict this dynamics can only be purely phenomenological, including data mining, model building, and parameter fitting [8, 9, with all their caveats [10, 11. However if two dynamical systems behave differently, it does not mean that the laws that govern their dynamics are different-the simplest example would be the quite different dynamics of two and three gravitating bodies of similar masses in empty space. And indeed if considering network dynamics we move from the small to large scale, we observe that preferential attachment [12 14 accurately describes the growth of many very different real networks [15-18. This observation raises two questions: (1) can preferential attachment be formulated within the canonical approach in physics, and if so, then (2) does the same approach apply to network dynamics at small scales?

Here we answer positively the first question by showing that preferential attachment can be fully described within the canonical formalism. That is, we show that the growth dynamics of networks evolving according to preferential attachment is Hamiltonian, and derive the explicit form of the Hamiltonian in the corresponding Hamilton's equations. This Hamiltonian turns out to be nearly identical to the Hamiltonian in the soft configuration model.

In the canonical formalism the dynamics of a system with canonical coordinates $(q, p)$ and Hamiltonian $\mathcal{H}(q, p, t)$, which is usually the total energy of the system, is described by Hamilton's equations

$$
\dot{q}=\frac{\partial \mathcal{H}}{\partial p}, \quad \dot{p}=-\frac{\partial \mathcal{H}}{\partial q} .
$$

The canonical approach has a long history of success in physics. All the fundamental interactions in nature are described by Euler-Lagrange or Hamilton's equations with different symmetry groups [19. The Einstein field equations in general relativity are Euler-Lagrange equations for the gravitational Einstein-Hilbert action, while the ADM formalism is the corresponding Hamiltonian formulation 20. Here we extend the canonical formalism to complex networks, and find the Hamiltonian describing the dynamics of growing networks in preferential attachment.

Most network models can be classified as either equilibrium models, which are the ensembles of graphs of fixed 
size, e.g. the Erdős-Rényi random graphs [21 23], or nonequilibrium models, in which networks grow with time, e.g. the preferential attachment model [12 14]. Equilibrium models are usually more amendable for analytical treatment, while non-equilibrium models better mimic the growth dynamics of real networks. In the special case of uncorrelated random graphs, there exist growing network models that produce equilibrium ensembles of graphs with an arbitrary degree distribution 24. In general however, the two types of models, growing and equilibrium, are very different, and so are the ensembles of random graphs that they define. A great number of works have studied equilibrium ensembles. A statistical theory of equilibrium correlated random graphs was developed in 25]. Review article 26] surveys important advances in the field of equilibrium network models. In particular, it discusses the structural properties of the graphs and topological phase transitions in equilibrium graph ensembles. An interesting "symbiotic" model, an equilibrium network model with fixed number of nodes and links which evolves using a local rewiring move, was studied in 27]. It was shown that if the graph Hamiltonian is chosen appropriately, then the networks generated by the model are scale-free. In all the prior works however, the graph Hamiltonians appear only in the equilibrium sense, i.e. as the graph energy proportional to the logarithm of the graph probability in the equilibrium ensemble. To the best of our knowledge no prior work has studied Hamiltonian dynamics of growing networks, where the graph Hamiltonian is the graph energy which defines the dynamics of growing graphs via Hamilton's equations (1).

Our starting point relies on recent results [28] establishing the special conditions under which there exists strong equivalence or duality between equilibrium and non-equilibrium (growing) graph ensembles $\left(\mathcal{G}_{N}, \mathbb{P}\right)$, where $\mathcal{G}_{N}$ is a set of graphs of size $N$, and $\mathbb{P}$ is a probability distribution on $\mathcal{G}_{N}$. Two network models or graph ensembles $\left(\mathcal{G}_{N}, \mathbb{P}_{1}\right)$ and $\left(\mathcal{G}_{N}, \mathbb{P}_{2}\right)$ are equivalent if they generate any graph $G \in \mathcal{G}_{N}$ with the same probability, i.e. $\mathbb{P}_{1}(G)=\mathbb{P}_{2}(G)$ for any $G$. We say that two ensembles are strongly equivalent, if they are equivalent for any $N$. Using these special conditions, we obtain an intermediate result, which is important in its own right. It gives an equilibrium formulation of preferential attachment. This formulation is useful because it allows, for the first time to the best of our knowledge, to explicitly calculate for any given graph $G$, e.g., a given real network, the probability $\mathbb{P}(G) \propto \exp [-H(G)]$ with which preferential attachment generates this graph, where $H(G)$ is the graph Hamiltonian (graph energy). This Hamiltonian turns out to be very similar to the Hamiltonian in the soft configuration model 29 31. Based on this intermediate result, we then derive the dynamic Hamiltonian $\mathcal{H}$ that governs the network evolution in preferential attachment.

Remarkably, the static Hamiltonian $H$ and its dynamic counterpart $\mathcal{H}$ turn out to be nearly identical. The only difference between the two is that exact node degrees in $H$ are replaced by their expected values in $\mathcal{H}$. We thus prove that preferential attachment and configuration model are in fact the same ensemble, or in other words, that preferential attachment generates nothing but random graphs with a given power-law degree distribution. One could in principle expect that to be true in view of several equilibrium(-like) approaches to preferential attachment 32 34]. In [32] it is shown how the hidden variable formalism introduced for equilibrium graph ensembles can be applied to the preferential attachment networks to derive their degree distribution and the correlation structure. In a similar spirit, [33] applies methods of statistical mechanics to compare equilibrium and non-equilibrium graph ensembles and, in particular, demonstrates that the degree distribution in preferential attachment is identical to that in the equilibrium ensemble of random trees. Recent work [34, among other results, proves that a minor modification of the preferential attachment model, called sequential preferential attachment, is identical to the equilibrium graph ensemble constructed using several Pólya urn processes. In this work we prove that the expectation that preferential attachment and configuration model are nearly equivalent is indeed correct.

The flow of logic in the paper, and a more detailed summary of the results are as follows. We begin with a recollection of basic facts concerning exponential random graph models (ERGMs) [8, 29, 35, 36] (Section II) and models of random graphs with hidden variables [32, 37. (Section III) that we will need in subsequent sections. In particular, in latter models, an equilibrium random graph ensemble is fully defined by a distribution $\rho(r)$ of hidden variables $r$ attached to nodes, and connection probability $p\left(r, r^{\prime}\right)$ between nodes, and two such ensembles are equivalent $\left(\mathbb{P}_{1}(G)=\mathbb{P}_{2}(G)\right)$, as soon their hidden variable distributions and connection probabilities are the same, $\rho_{1}(r)=\rho_{2}(r)$ and $p_{1}\left(r, r^{\prime}\right)=p_{2}\left(r, r^{\prime}\right)$. Random graphs with hidden variables are not ERGs per se, but they are collections of ERGMs with fixed values of hidden variables playing the role of Lagrange multipliers.

We then briefly discuss soft preferential attachment (SPA, Section IV). SPA is different from standard preferential attachment (PA) in only that in PA new links attach to existing node $i$ with probability proportional to its degree $k_{i}$, while in SPA new links attach to existing node $i$ with probability proportional to its expected degree $\kappa_{i}$. The idea behind the SPA definition is to assign to each new node $j$ a hidden variable $r_{j} \sim \log j$, and then connect $j$ to existing node $i$ with certain probability that depends only on the current values of $j$ 's and $i$ 's hidden variables $r_{j}$ and $r_{i}$. The words current values appear here because the values of these hidden variables $r$ need to be updated as the network grows, and the combination of this update rule and connection probability are such that new nodes do indeed connect to existing nodes with probability proportional to their current expected degrees, so that we do have SPA. The key point behind SPA is that it has a coupled dynamics of hidden vari- 
ables $r_{i}$ and expected degrees $\kappa_{i}$, both growing functions of the network size $N=1,2, \ldots$ or "cosmological time" $t \sim \log N$.

Next, in Section V] we show that SPA is an ERGM, which is asymptotically $(N \gg 1)$ identical to the soft configuration model (SCM) for sparse graphs with average degree $\bar{k} \ll N$. We do this in steps. We first recall, in Section $\mathrm{VA}$, that the SCM is an ERGM with Hamiltonian $H(G)=\sum_{i} k_{i} r_{i}+C$, where $k_{i}$ is the degree of node $i$ in graph $G, r_{i}$ is the Lagrange multiplier fixing the expected values of $k_{i}$ in this ERG ensemble to some value $\left\langle k_{i}\right\rangle$, and $C$ are some additional terms. This ensemble is an equilibrium ensemble of random graphs with a given sequence of expected degrees $\left\langle k_{i}\right\rangle$. If this sequence is power-law-distributed, then the sequence of $r_{i} \mathrm{~s}$ is exponentially distributed. If this $r$-sequence is not fixed but sampled, for each graph, from a fixed exponential distribution $\rho(r)$, then the resulting SCM, is an ERGM with hidden variables $r$ of random graphs with a given expected power-law degree distribution. The next two most technical sections deal with certain cosmetic adjustments to $\mathrm{SCM}\left(\mathrm{SCM}^{+}\right.$, Section $\left.\mathrm{VB}\right)$ and SPA ( $\widetilde{\mathrm{SPA}}$, Section VC, and in Section VD we show that after these adjustments, the two models $\left(\mathrm{SCM}^{+}\right.$and $\left.\widetilde{\mathrm{SPA}}\right)$ are asymptotically equivalent, and derive their equilibrium ERG Hamiltonians $H$ in Section VE

Finally, in Section VI, we turn back to the dynamics of SPA, and pose the question: is there a dynamic Hamiltonian $\mathcal{H}\left(\kappa_{i}, r_{i}, t\right)$ such that the dynamics of expected degrees $\kappa_{i}(t)$ and hidden variables $r_{i}(t)$ in SPA is the solution of Hamilton's equation with this Hamiltonian $\mathcal{H}$ ? We answer this question positively by first deriving the exact dynamics of $\kappa_{i} \mathrm{~s}$ in SPA and SPA (Sections VIA and VIB, and then finding a whole family of Hamiltonians that provide a solution to the question above (Section VIC). This family is parameterized by arbitrary functions $\xi_{i}(t)$, and we show in the same section that $\xi_{i}(t)$ can be selected such that the resulting dynamic Hamiltonian $\mathcal{H}$ in $\widetilde{\mathrm{SPA}}$, evaluated on the solution of Hamilton's equation, is equal, for any value of graph size $N$, to the equilibrium $\mathrm{SCM}^{+}$Hamiltonian $H$ in Section VE, upon substitution $\kappa_{i}(N)=k_{i}$.

\section{EXPONENTIAL RANDOM GRAPHS}

The exponential random graph model (ERGM) [8, 29, 35, 36] is one of the most popular and well-studied equilibrium network models, also known as the $p^{*}$ model in the social network research community [38 40]. ERGM is a graph ensemble $\left(\mathcal{G}_{N}, \mathbb{P}\right)$, where $\mathcal{G}_{N}$ is the set of all simple graphs (i.e. undirected graphs without self-loops or multi-edges) on $N$ nodes, and $\mathbb{P}$ is the probability distribution on $\mathcal{G}_{N}$ that maximizes the Gibbs entropy

$$
S(\mathbb{P})=-\sum_{G \in \mathcal{G}_{N}} \mathbb{P}(G) \ln \mathbb{P}(G) \rightarrow \max
$$

subject to the constraints

$$
\left\langle x_{i}\right\rangle=\bar{x}_{i}, \quad i=1, \ldots, r .
$$

The $x_{i}$ in the above relation are certain graph properties (e.g. number of edges or number of triangles) often referred to as the graph "observables", $\bar{x}_{i}$ are the prescribed expected values of these observables in the model, and $\langle\cdot\rangle$ denotes the expectation with respect to $\mathbb{P}$. Intuitively, an ERGM is a "maximally random" ensemble of graphs with fixed values $\bar{x}_{i}$ for certain ensemble averages $\left\langle x_{i}\right\rangle$. Mathematically, the maximization of randomness corresponds to the maximization of the entropy (2). Constraining the expected rather than exact values of graph observables relaxes the topological conditions on the network and makes the model amenable to analytical treatment.

The constrained optimization problem (2) and (3) can be solved by the standard method of Lagrange multipliers, and it has the following explicit solution [29]

$$
\mathbb{P}(G)=\frac{e^{-H(G)}}{Z},
$$

where

$$
Z=\sum_{G \in \mathcal{G}_{N}} e^{-H(G)}
$$

is the partition function, i.e. the normalizing constant for distribution (4), and

$$
H(G)=\sum_{i=1}^{r} \theta_{i} x_{i}(G)
$$

is the graph Hamiltonian, i.e. the energy of microstate $G$ in the equilibrium Boltzmann distribution (4). The parameters $\theta_{i}$ are the Lagrange multipliers ("auxiliary fields") coupled to observables $x_{i}$. They are determined by the following system of $r$ equations

$$
\frac{\partial F}{\partial \theta_{i}}=\bar{x}_{i}, \quad i=1, \ldots, r,
$$

where $F=-\ln Z$ is the free energy. The ERGM distribution (4) is thus fully determined by the observables $x_{i}$ and their expected values $\bar{x}_{i}$. In [41, the ERGM is extended to exponential random simplicial complexes.

As an example of an ERGM, which we will refer to in Section $\mathrm{VA}$, consider the edge-independent random graph (EIRG) model. In this case, the graph observables are the graph edges: $x_{i j}=a_{i j}$, where $a=\left(a_{i j}\right)$ is the adjacency matrix of $G \in \mathcal{G}_{N}$. The constrains (3) are then

$$
\left\langle a_{i j}\right\rangle=p_{i j}, \quad i<j, \quad i, j=1, \ldots, N,
$$

where $0 \leq p_{i j} \leq 1$, and the Hamiltonian is

$$
H(G)=\sum_{i<j} \theta_{i j} a_{i j}
$$


Unlike many other examples, the partition function $Z$ can be calculated exactly for the EIRG model [29]:

$$
Z=\prod_{i<j}\left(1+e^{-\theta_{i j}}\right)
$$

The relationship between the Lagrange multipliers $\theta_{i j}$ and the model parameters $p_{i j}$ follows from (7)

$$
p_{i j}=\frac{1}{1+e^{\theta_{i j}}} .
$$

Knowing the partition function allows to find the corresponding ERGM distribution (4)

$$
\mathbb{P}(G)=\prod_{i<j} p_{i j}^{a_{i j}}\left(1-p_{i j}\right)^{1-a_{i j}}
$$

This expression immediately suggests how to generate graphs from the maximum-entropy ensemble $\left(\mathcal{G}_{N}, \mathbb{P}\right)$ : connect every pair $(i, j)$ of distinct nodes $i \neq j, i, j=$ $1, \ldots, N$, independently at random with probability $p_{i j}$. We remark that (11) is nothing but the Fermi-Dirac distribution, where the Lagrange multiplier $\theta_{i j}$ is interpreted as the energy of the "single-particle" state $(i, j)$. Throughout the paper, we will often use the so-called classical limit for the Fermi-Dirac distribution, i.e. if the energy $\theta_{i j}$ is large, then $p_{i j} \approx e^{-\theta_{i j}}$.

\section{RANDOM GRAPHS WITH HIDDEN VARIABLES}

Random graphs with hidden variables [32, 37] are ensembles of random graphs in which graphs are generated (or sampled) as follows. Each node $i=1, \ldots, N$ is first assigned a hidden variable $r_{i}$, sampled from the probability distribution $\rho(r)$, and then each pair of nodes $(i, j)$ is connected with probability $p_{i j}=p\left(r_{i}, r_{j}\right)$. Since the hidden random variables are independent, the probability $\mathbb{P}(G)$ of graph $G$ in the ensemble $\left(\mathcal{G}_{N}, \mathbb{P}\right)$ is

$$
\begin{aligned}
\mathbb{P}(G) & =\int \mathbb{P}(G \mid \mathbf{r}) \rho(\mathbf{r}) d \mathbf{r} \\
& =\int \prod_{i>j} p_{i j}^{a_{i j}}\left(1-p_{i j}\right)^{1-a_{i j}} \prod_{i=1}^{N} \rho\left(r_{i}\right) d r_{i}
\end{aligned}
$$

where $\left(a_{i j}\right)$ is $G$ 's adjacency matrix and $\mathbf{r}=\left(r_{1}, \ldots, r_{N}\right)$. This equilibrium graph ensemble is thus fully defined by two functions: the hidden variable PDF $\rho(r)$ and the connection probability function $p\left(r, r^{\prime}\right)$.

In equilibrium statistical mechanics, two ensembles are equivalent if their state occupation probabilities are the same for any state. Similarly, two graph ensembles $\left(\mathcal{G}_{N}, \mathbb{P}_{1}\right)$ and $\left(\mathcal{G}_{N}, \mathbb{P}_{2}\right)$ are equivalent if they generate any graph $G \in \mathcal{G}_{N}$ with the same probability:

$$
\mathbb{P}_{1}(G)=\mathbb{P}_{2}(G)
$$

It follows from Eq. 13 that two ensembles of random graphs with hidden variables are equivalent if their hidden variable distributions and connection probabilities are the same:

$$
\begin{aligned}
\rho_{1}(r) & =\rho_{2}(r), \\
p_{1}\left(r, r^{\prime}\right) & =p_{2}\left(r, r^{\prime}\right) .
\end{aligned}
$$

Random graphs with hidden variables are closely related to exponential random graphs. If we sample all hidden variables $r_{i}$ from $\rho(r)$ just once, and then fix them, then the probability of graph $G$ in the ensemble with these fixed $r_{i}$ s is given by $(12)$, with $p_{i j}=p\left(r_{i}, r_{j}\right)$. Rewriting these $p_{i j}$ s as $1 /\left(1+e^{\theta_{i j}}\right)$ makes the ensemble manifestly identical to the EIRG ensemble with Lagrange multipliers $\theta_{i j}$. Therefore one can think of graphs with unfixed (sampled) hidden variables as a collection EIRGs with "randomized" Lagrange multipliers sampled from a fixed distribution.

\section{SOFT PREFERENTIAL ATTACHMENT}

Our first goal is to represent the preferential attachment (PA) model as an ERGM. The original formulation of PA [12, where a new node connects to an existing node with probability proportional to its degree, is very intuitive, but not convenient for this purpose. Instead, we will use a hidden variable formulation of PA. It was shown in 42 that PA can be formulated as a hidden variable model, which generates growing networks up to some size $N$ with average degree $\bar{k}$ and power-law exponent $\gamma \geq 2$, as follows. For each new node $i=1, \ldots, N$ :

1. Assign to node $i$ hidden variable

$$
r_{i}=\ln i
$$

2. Update the values of hidden variables of all existing nodes $j<i$ by setting

$$
\begin{aligned}
r_{j}(i) & =\beta r_{j}+(1-\beta) r_{i}, \text { where } \\
\beta & =\frac{1}{\gamma-1} .
\end{aligned}
$$

3. Connect node $i$ to each existing node $j<i$ with probability

$$
p_{i j}=\frac{1}{1+e^{r_{j}(i)+r_{i}-R_{i}}},
$$

where

$$
R_{i}=r_{i}-\ln \frac{1-e^{-(1-\beta) r_{i}}}{m(1-\beta)} \text { and } m=\frac{\bar{k}}{2} .
$$

In large sparse networks, where $r_{i}$ is large and $\beta<1$, the linking probability

$$
p_{i j} \approx m(1-\beta) e^{-r_{j}(i)} \propto e^{-r_{j}(i)}=i^{-1}\left(\frac{i}{j}\right)^{\beta} .
$$


In Section VIA, we show that the expected degree of node $j$ at time $i$ is

$$
\kappa_{j}(i)=\frac{m(1-\beta)}{\beta}\left(\frac{i}{j}\right)^{\beta}+\frac{m(2 \beta-1)}{\beta},
$$

and therefore, the probability that $i$ connects to $j$ is approximately a liner function of $j$ 's expected degree $\kappa_{j}(i)$. We note that Eq. (23) holds for $\beta<1$, i.e., $\gamma>2$. The corresponding relation for the limit $\beta \rightarrow 1(\gamma \rightarrow 2)$ is derived in Appendix A.1. We refer to the hidden variable formulation of PA as the soft preferential attachment (SPA) model. Figure 1(a) shows a doubly logarithmic plot of the empirical degree distribution in a network generated by SPA along with the fitted power-law distribution.

A conceptually similar formulation of preferential attachment as an equilibrium network model with hidden variables was first introduced in 32, where the hidden variable $r_{i}$ of node $i$ is simply its injection time, $r_{i}=i$. For technical reasons that will become apparent in the next section, here we define $r_{i}=\ln i$, so that $r_{i}$ can be identified with the cosmological time of birth of node $i$ [42, 43].

\section{SPA AS AN ERGM}

Let $\left(\mathcal{G}_{N}, \mathbb{P}_{\mathrm{SPA}}\right)$ be the ensemble of graphs induced by SPA, where $\mathbb{P}_{\text {SPA }}(G)$ is the probability that SPA generates $G \in \mathcal{G}_{N}$. In this section we show that

$$
\mathbb{P}_{\text {SPA }}(G) \propto e^{-H_{\text {SPA }}(G)},
$$

where the SPA Hamiltonian $H_{\mathrm{SPA}}$ is intimately related to the Hamiltonian in the soft configuration model.

\section{A. Soft Configuration Model}

The soft configuration model (SCM) 29 31] is an ERGM, where graph observables are node degrees $k_{i}$, $i=1, \ldots, N$. The model has various equivalent formulations, and, in particular, SCM appears as a special degenerate case of the equilibrium hyperbolic model [43] in a certain limiting parameter regime. This formulation also belongs to the wide class of network models with hidden variables. Specifically, the SCM formulation in [43] generates equilibrium networks of size $N$ with average degree $\bar{k}$ and power-law exponent $\gamma>2$, as follows:

1. For $i=1, \ldots, N$, assign to node $i$ hidden variable

$$
r_{i} \sim \rho_{\mathrm{SCM}}(r) \approx \alpha e^{\alpha\left(r-R_{\mathrm{SCM}}\right)}, \quad 0 \leq r \leq R_{\mathrm{SCM}}
$$

where $\alpha=\beta^{-1}=\gamma-1$ and

$$
R_{\mathrm{SCM}}=\ln \frac{N}{\bar{k}(1-\beta)^{2}} .
$$

2. Connect nodes $i$ and $j, j \neq i$, with probability

$$
p_{i j}=\frac{1}{1+e^{r_{i}+r_{j}-R_{\mathrm{SCM}}}} .
$$

A more familiar (but less convenient for our purposes) formulation of the SCM is obtained by the change of hidden variables $h=h_{0} e^{R_{\mathrm{SCM}}-r}, h_{0}=\bar{k}(1-\beta)$. The hidden variable $h$ has then the power-law distribution $h \sim \rho(h) \propto h^{-\gamma}$ and the connection probability is $p_{i j}=1 /\left(1+N \bar{k} / h_{i} h_{j}\right)[28$, 44, 45]. Comparing the connection probabilities in EIRG (11) and SCM (27), we readily obtain that the Lagrange multiplier $\theta_{i j}$ in SCM is

$$
\theta_{i j}=r_{i}+r_{j}-R_{\mathrm{SCM}}
$$

The SCM Hamiltonian is then

$$
\begin{aligned}
H_{\mathrm{SCM}}(G) & =\sum_{i<j} \theta_{i j} a_{i j}=\sum_{i<j}\left(r_{i}+r_{j}-R_{\mathrm{SCM}}\right) a_{i j} \\
& =\sum_{i=1}^{N} k_{i} r_{i}-M R_{\mathrm{SCM}}
\end{aligned}
$$

where $k_{i}$ is the degree of node $i$ and $M$ is the total number of edges in the graph $G$.

Figure 1(a) shows the degree distribution in a network generated by SCM, which is identical to the degree distribution in an SPA network generated with the same parameters. As expected, both are power-laws with exponent $\gamma$. Figure 1 (b) shows the empirical distributions of the hidden variables $\rho_{\mathrm{SPA}}(r)$ and $\rho_{\mathrm{SCM}}(r)$ in the generated networks. Although both distributions are highly skewed to the right, there is a clear discrepancy between them. In the next section we fix this discrepancy by introducing a shifted SCM model, which is strongly equivalent to SCM, but has the same distribution of hidden variables as SPA.

\section{B. Shifted Soft Configuration Model}

While the hidden variables in SCM are random, in SPA they are deterministic. Nevertheless we can readily overcome this technical obstruction that hinders the comparison of hidden variable distributions in the two models.

Let $r_{*}(N)$ denote one of the hidden variables $r_{1}(N), \ldots, r_{N}(N)$ in SPA chosen uniformly at random 

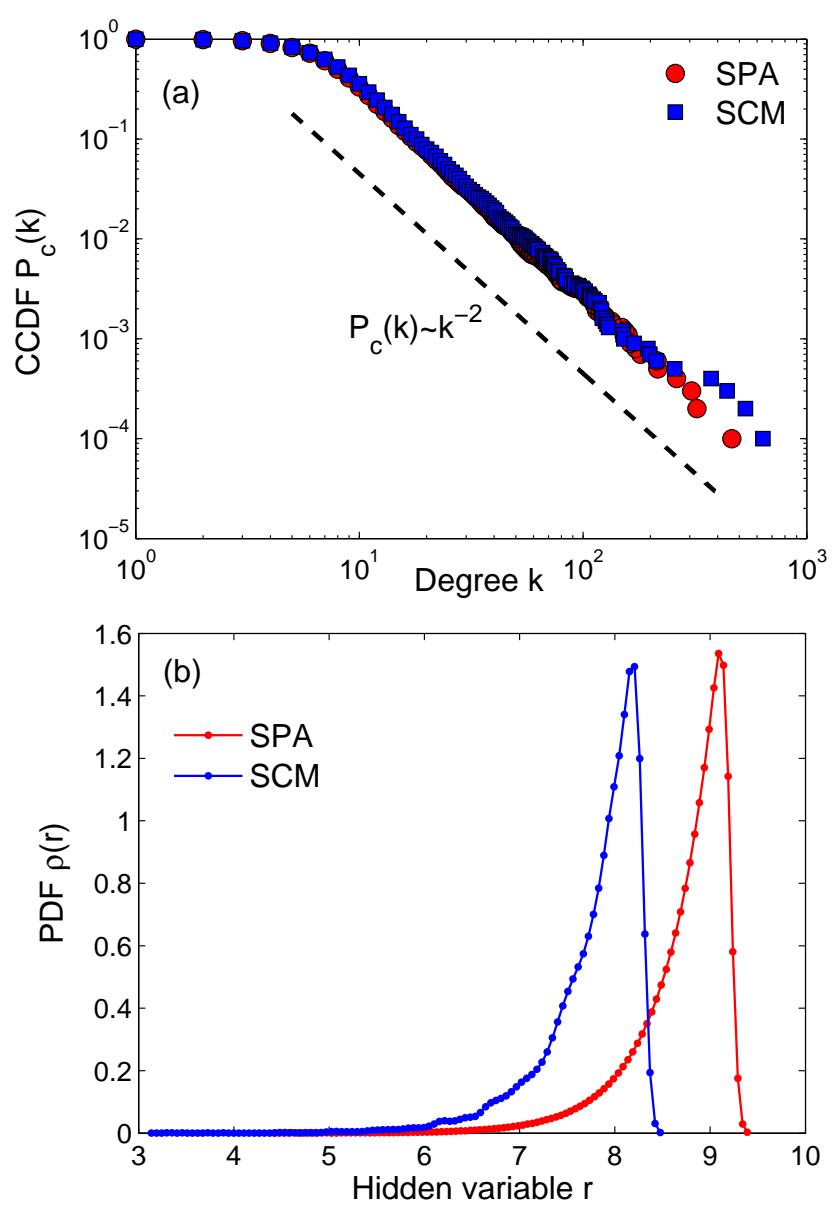

FIG. 1: SPA and SCM networks. Panel (a) shows the empirical complementary cumulative degree distribution functions $(\mathrm{CCDF}) P_{c}(k)=\sum_{k^{\prime}>k} \mathbb{P}\left(k^{\prime}\right)$ for two networks of size $N=10^{4}$ generated by SPA and SCM with $\bar{k}=10$ and $\gamma=3$, and the corresponding power-law fit. As expected, $P_{c}(k) \sim k^{-\gamma+1}$. Panel (b) shows the empirical probability density functions (PDF) of the hidden variables $r_{i}$ in these two networks.

at time $N \gg 1$. The $\mathrm{CDF}$ of $r_{*}(N)$ is then

$$
\begin{aligned}
F_{r_{*}(N)}(r) & =\mathbb{P}\left(r_{*}(N) \leq r\right)=\frac{\left|\left\{i: r_{i}(N) \leq r\right\}\right|}{N} \\
& =\frac{|\{i: \beta \ln i+(1-\beta) \ln N \leq r\}|}{N} \\
& =\frac{\left|\left\{i: i \leq e^{\frac{r}{\beta}-\frac{1-\beta}{\beta} \ln N}\right\}\right|}{N}=\frac{1}{N} \sum_{i \leq e^{\frac{r}{\beta}-\frac{1-\beta}{\beta} \ln N}} 1 \text { (30) } \\
& \approx \frac{1}{N} \int_{0}^{e^{\frac{r}{\beta}-\frac{1-\beta}{\beta} \ln N} 1 d i=e^{\frac{r-\ln N}{\beta}} .}
\end{aligned}
$$

When $N$ is large, the SPA hidden variables $r_{1}(N), \ldots, r_{N}(N)$ can be viewed as being approximately i.i.d. samples from $F_{r_{*}(N)}(r)$. This distribution has the following PDF

$$
\rho_{\mathrm{SPA}}(r)=\frac{d}{d r} F_{r_{*}(N)}(r)=\frac{1}{\beta} e^{\frac{r-\ln N}{\beta}},
$$

which is structurally similar to the distribution of hidden variables in $\mathrm{SCM}$

$$
\begin{aligned}
\rho_{\mathrm{SCM}}(r) & \approx \alpha e^{\alpha\left(r-R_{\mathrm{SCM}}\right)}=\frac{1}{\beta} e^{\frac{r-\ln N+\sigma}{\beta}}, \text { where } \\
\sigma & =\ln \bar{k}(1-\beta)^{2} .
\end{aligned}
$$

It is readily verifiable that the approximate supports of $\rho_{S P A}(r)$ and $\rho_{S C M}(r)$, i.e. segments that contain almost all probability mass of these distributions, are

$$
\begin{aligned}
\operatorname{supp} \rho_{P A} & =[(1-\beta) \ln N, \ln N], \\
\operatorname{supp} \rho_{S C M} & =[(1-\beta) \ln N-\sigma, \ln N-\sigma] .
\end{aligned}
$$

Therefore, it immediately follows from (31)- 35 that $\rho_{S P A}(r)$ is obtained from $\rho_{S C M}(r)$ by translation by $\sigma$. This motivates the shifted SCM model, denoted $\mathrm{SCM}^{+}$, which generates networks of size $N$ with average degree $\bar{k}$ and power-law exponent $\gamma>2$, as follows:

1. For $i=1, \ldots, N$, assign to node $i$ hidden variable $r_{i}^{+}$by, first, sampling $r_{i} \sim \rho_{\mathrm{SCM}}(r)$, and then shifting $r_{i}^{+}=r_{i}+\sigma$, where $\sigma=\ln \bar{k}(1-\beta)^{2}$.

2. Connect nodes $i$ and $j, j \neq i$, with probability

$$
p_{i j}=\frac{1}{1+e^{r_{i}^{+}+r_{j}^{+}-R_{\mathrm{SCM}}+}},
$$

where $R_{\mathrm{SCM}^{+}}$is given by 39 .

By construction, the distributions of hidden variables in $\mathrm{SCM}^{+}$and SPA are identical

$$
\rho_{\mathrm{SCM}^{+}}(r)=\rho_{\mathrm{SCM}}(r-\sigma)=\rho_{\mathrm{SPA}}(r) .
$$

To make $\mathrm{SCM}^{+}$equivalent to $\mathrm{SCM}$, we need to choose $R_{\mathrm{SCM}^{+}}$appropriately. If two nodes have hidden variables $r$ and $r$ in SCM, then in $\mathrm{SCM}^{+}$the values of these hidden variables are $r^{+}=r+\sigma$ and $\dot{r}^{+}=\dot{r}+\sigma$. The two models will be strongly equivalent, i.e. will generate graphs $G \in \mathcal{G}_{N}$ with equal probabilities $\mathbb{P}_{\mathrm{SCM}}(G)=$ $\mathbb{P}_{\mathrm{SCM}^{+}}(G)$, if the connection probabilities $p_{\mathrm{SCM}}\left(r, r^{\prime}\right)$ and $p_{\mathrm{SCM}^{+}}\left(r^{+}, r^{+}\right)$are the same. This leads to the following equation for $R_{\mathrm{SCM}^{+}}$

$$
r+\dot{r}-R_{\mathrm{SCM}}=r^{+}+\dot{r}^{+}-R_{\mathrm{SCM}^{+}} .
$$

Therefore,

$$
R_{\mathrm{SCM}^{+}}=\ln N+\sigma=\ln N \bar{k}(1-\beta)^{2} .
$$

It is convenient to work with SPA and $\mathrm{SCM}^{+}$(instead of SCM) since not only the degree distributions in the networks generated by these two models match, but also the distributions of hidden variables are the same. Our next step is to adjust SPA so that it becomes strongly equivalent to $\mathrm{SCM}^{+}$(and, therefore, to $\mathrm{SCM}$ ). 


\section{Bridging SPA and $\mathrm{SCM}^{+}$}

Matching degree distributions is a necessary but, of course, not sufficient condition for model equivalence. In SPA, a link between nodes $i$ and $j<i$ may appear only at time $i$ upon the birth of the younger node. We refer to such links - appearing at time $i$ and connecting new node $i$ to already existent nodes — as "external" links. We make the SPA model equivalent to $\mathrm{SCM}^{+}$by also allowing "internal" links that appear at time $i$ and connect old nodes $a$ and $b$, where $a, b<i$. Namely, we define the model $\widetilde{\mathrm{SPA}}$ that generates growing networks up to some size $N$ with average degree $\bar{k}$ and power-law exponent $\gamma>2$, as follows. For each new node $i=1, \ldots, N$ :

1. Assign to node $i$ hidden variable $r_{i}=\ln i$.

2. Update the values of hidden variables of all existing nodes $j<i$ by setting $r_{j}(i)=\beta r_{j}+(1-\beta) r_{i}$, where $\beta=\frac{1}{\gamma-1}$.

3. Connect node $i$ to each existing node $j<i$ with probability

$$
p_{i j}^{\text {ext }}=\frac{1}{1+e^{r_{j}(i)+r_{i}-R_{i}^{\text {ext }}}},
$$

where

$$
R_{i}^{\mathrm{ext}}=r_{i}-\ln \frac{1-e^{-(1-\beta) r_{i}}}{m_{\mathrm{ext}}(1-\beta)},
$$

and $m_{\text {ext }}$ given by 46 .

4. Connect each pair of existing nodes $a, b<i$ with probability

$$
p_{a b}^{\mathrm{int}}(i)=\frac{1}{1+e^{r_{a}(i)+r_{b}(i)-R^{\mathrm{int}}}},
$$

where

$$
R^{\text {int }}=\ln m_{\text {int }}(1-\beta),
$$

and $m_{\text {int }}$ given by 50 .

In Step 4, we scan all pairs of existing nodes and attempt to connect even those nodes which are already connected. The $\widetilde{\text { SPA }}$ model thus allows multi-edges. In large sparse $(\bar{k} \ll N)$ networks, however, the proportion of multiedges is small. For example, the expected ratio of multiedges in $\widetilde{\mathrm{SPA}}$ networks of size $N=10^{2}, 10^{3}$, and $10^{4}$ with $\bar{k}=10$ and $\gamma=2.5$ is, respectively, $7 \%, 4 \%$ and $2 \%$. We can therefore ignore the multi-edge effect. The choices for $m_{\text {ext }}$ and $m_{\text {int }}$ (Eqs. (46) and (50) ) are explained below.

First, a necessary (but not sufficient) condition for the equivalence of two models is that the expected minimum degrees in the two models must be the same. The expected minimum degree in large networks generated by SCM (and therefore by $\mathrm{SCM}^{+}$) is $\bar{k}(1-\beta)$ [3]. Thus, we have the following condition

$$
\left\langle k_{\min }\right\rangle_{\widetilde{\mathrm{SPA}}}=\bar{k}(1-\beta) \text {. }
$$

Let us now compute the expected degree of a new node $i$ upon its birth in $\widetilde{\mathrm{SPA}}$. For large $i, R_{i}^{\text {ext }} \approx r_{i}+\ln m_{\text {ext }}(1-$ $\beta$ ), and using the classical limit for the Fermi-Dirac distribution, we get

$$
\begin{aligned}
\kappa_{i}(i) & =\sum_{j<i} p_{i j}^{\mathrm{ext}} \approx \int_{0}^{i} \frac{d j}{1+e^{r_{j}(i)+r_{i}-R_{i}^{\mathrm{ext}}}} \\
& \approx \int_{0}^{i} \frac{d j}{1+e^{\beta r_{j}+(1-\beta) r_{i}-\ln m_{\mathrm{ext}}(1-\beta)}} \\
& =\int_{0}^{i} \frac{d j}{1+\frac{j^{\beta} i^{1-\beta}}{m_{\mathrm{ext}}(1-\beta)}} \approx \frac{m_{\mathrm{ext}}(1-\beta)}{i^{1-\beta}} \int_{0}^{i} j^{-\beta} d j \\
& =m_{\mathrm{ext}} .
\end{aligned}
$$

Every new node thus establishes on average $m_{\text {ext }}$ links, and, as time goes, its degree may only increase. This means that $m_{\text {ext }}$ is the expected minimum degree in $\widetilde{\mathrm{SPA}}$, $\left\langle k_{\min }\right\rangle_{\widetilde{\mathrm{SPA}}}=m_{\mathrm{ext}}$, and therefore

$$
m_{\mathrm{ext}}=\bar{k}(1-\beta)
$$

Another necessary condition (that helps to determine $m_{\text {int }}$ ) for the equivalence between $\widetilde{\mathrm{SPA}}$ and $\mathrm{SCM}^{+}$is that the expected average degrees in both models must be the same. That is, if in $\mathrm{SCM}^{+}$the expected average degree $\langle\bar{k}\rangle_{\mathrm{SCM}^{+}}$equals $\bar{k}$, then we must have

$$
\langle\bar{k}\rangle_{\widetilde{\mathrm{SPA}}}=\bar{k}
$$

Let $\bar{L}_{i}^{\text {int }}$ denote the expected number of internal links generated at time $i$. Then, the expected total number of links generated at time $i$ is $m_{\text {ext }}+\bar{L}_{i}^{\text {int }}$, and the expected average degree in the network is given by

$$
\langle\bar{k}\rangle_{\widetilde{\mathrm{SPA}}} \approx \frac{2}{N} \int_{0}^{N}\left(m_{\mathrm{ext}}+\bar{L}_{i}^{\mathrm{int}}\right) d i .
$$

For large $i$,

$$
\begin{aligned}
\bar{L}_{i}^{\mathrm{int}} & =\sum_{a<i} \sum_{b<a} p_{a b}^{\mathrm{int}}(i) \approx \int_{0}^{i} \int_{0}^{a} \frac{d b d a}{1+e^{r_{a}(i)+r_{b}(i)-R^{\mathrm{int}}}} \\
& =\int_{0}^{i} \int_{0}^{a} \frac{d b d a}{1+e^{\beta r_{a}+\beta r_{b}+2(1-\beta) r_{i}-\ln m_{\mathrm{int}}(1-\beta)}} \\
& =\int_{0}^{i} \int_{0}^{a} \frac{d b d a}{1+\frac{a^{\beta} b^{\beta} i^{2(1-\beta)}}{m_{\mathrm{int}}(1-\beta)}} \\
& \approx \frac{m_{\mathrm{int}}(1-\beta)}{i^{2(1-\beta)}} \int_{0}^{i} \int_{0}^{a} a^{-\beta} b^{-\beta} d b d a \\
& =\frac{m_{\mathrm{int}}}{2(1-\beta)} .
\end{aligned}
$$

Combining (46)-49, we get $m_{\text {int }}$

$$
m_{\text {int }}=\bar{k}(1-\beta)(2 \beta-1),
$$

which is positive if $\beta \in(1 / 2,1)$, or, equivalently, $\gamma \in$ $(2,3)$. We note that $2<\gamma<3$ is exactly the range of 
power-law exponents empirically observed in most real networks [46. Figure 2 shows the perfect match between the distributions of node degrees and hidden variables in $\widetilde{\mathrm{SPA}}$ and $\mathrm{SCM}^{+}$networks with $\gamma=2.5$. Recall that the match between the hidden variable distributions is the first condition (15) for two ensembles of random graphs with hidden variables to be equivalent.

We note that as $\beta \rightarrow 1 / 2$, or, equivalently, $\gamma \rightarrow 3$, $\widetilde{\mathrm{SPA}}$ becomes manifestly identical to SPA. Indeed, in this case,

$$
m_{\mathrm{ext}} \rightarrow m=\frac{\bar{k}}{2} \quad \text { and } \quad m_{\mathrm{int}} \rightarrow 0
$$

which means

$$
R^{\text {int }} \rightarrow-\infty \quad \text { and } \quad p_{a b}^{\text {int }}(i) \rightarrow 0, \forall i
$$

The other limiting case $\beta \rightarrow 1(\gamma \rightarrow 2)$ is analyzed in Appendix A.1.

It is important to realize that by choosing $m_{\text {ext }}$ and $m_{\text {int }}$ according to 46 and (50) we only satisfied two necessary conditions, but we did not actually prove that $\widetilde{\mathrm{SPA}}$ is equivalent to $\mathrm{SCM}^{+}$. We prove this in the next section.

\section{D. $\widetilde{\text { SPA }}$ and $\mathrm{SCM}^{+}$are strongly equivalent}

Since the distributions of hidden variables in $\widetilde{\mathrm{SPA}}$ and $\mathrm{SCM}^{+}$are the same, i.e. $\rho_{\widetilde{\mathrm{SPA}}}(r)=\rho_{\mathrm{SCM}^{+}}(r)$, to prove the strong equivalence between the two models, we need to show that the connection probabilities in $\widetilde{\mathrm{SPA}}$ and $\mathrm{SCM}^{+}$are also the same. More precisely, if at time $N$ the values of hidden variables of nodes $j$ and $i>j$ in $\widetilde{\mathrm{SPA}}$ are $r$ and $r>r$, then the probability that these two nodes are connected $p_{\widetilde{\mathrm{SPA}}}(i, j)$ must be equal to the connection probability $p_{\mathrm{SCM}^{+}}\left(r, r^{\prime}\right)$ of nodes with hidden variables $r$ and $r$ in $\mathrm{SCM}^{+}$. In what follows, we compute these probabilities in large sparse graphs $(N \gg 1, \bar{k} \ll N)$ and show that they indeed coincide. Throughout this section we assume that $\beta \in(1 / 2,1)(\gamma \in(2,3))$.

Let $p_{\mathrm{SPA}}^{*}(i, j)$ denote the probability that nodes $i$ and $j$ are not connected in $\widetilde{\mathrm{SPA}}$, then

$$
\begin{aligned}
& p_{\widetilde{\mathrm{SPA}}}(i, j)=1-p_{\mathrm{SPA}}^{*}(i, j) \\
& =1-\left(1-p_{i j}^{\mathrm{ext}}\right)\left(1-p_{i j}^{\mathrm{int}}(i+1)\right) \ldots\left(1-p_{i j}^{\mathrm{int}}(N)\right) \\
& \approx p_{i j}^{\mathrm{ext}}+\sum_{s>i}^{N} p_{i j}^{\mathrm{int}}(s) \approx p_{i j}^{\mathrm{ext}}+\int_{i}^{N} p_{i j}^{\mathrm{int}}(s) d s .
\end{aligned}
$$

Let us compute the integral first

$$
\begin{gathered}
\int_{i}^{N} p_{i j}^{\mathrm{int}}(s) d s=\int_{i}^{N} \frac{d s}{1+e^{r_{i}(s)+r_{j}(s)-R^{\mathrm{int}}}} \\
\quad=\int_{i}^{N} \frac{d s}{1+e^{\beta r_{i}+\beta r_{j}+2(1-\beta) \ln s-\ln m_{\mathrm{int}}(1-\beta)}} .
\end{gathered}
$$
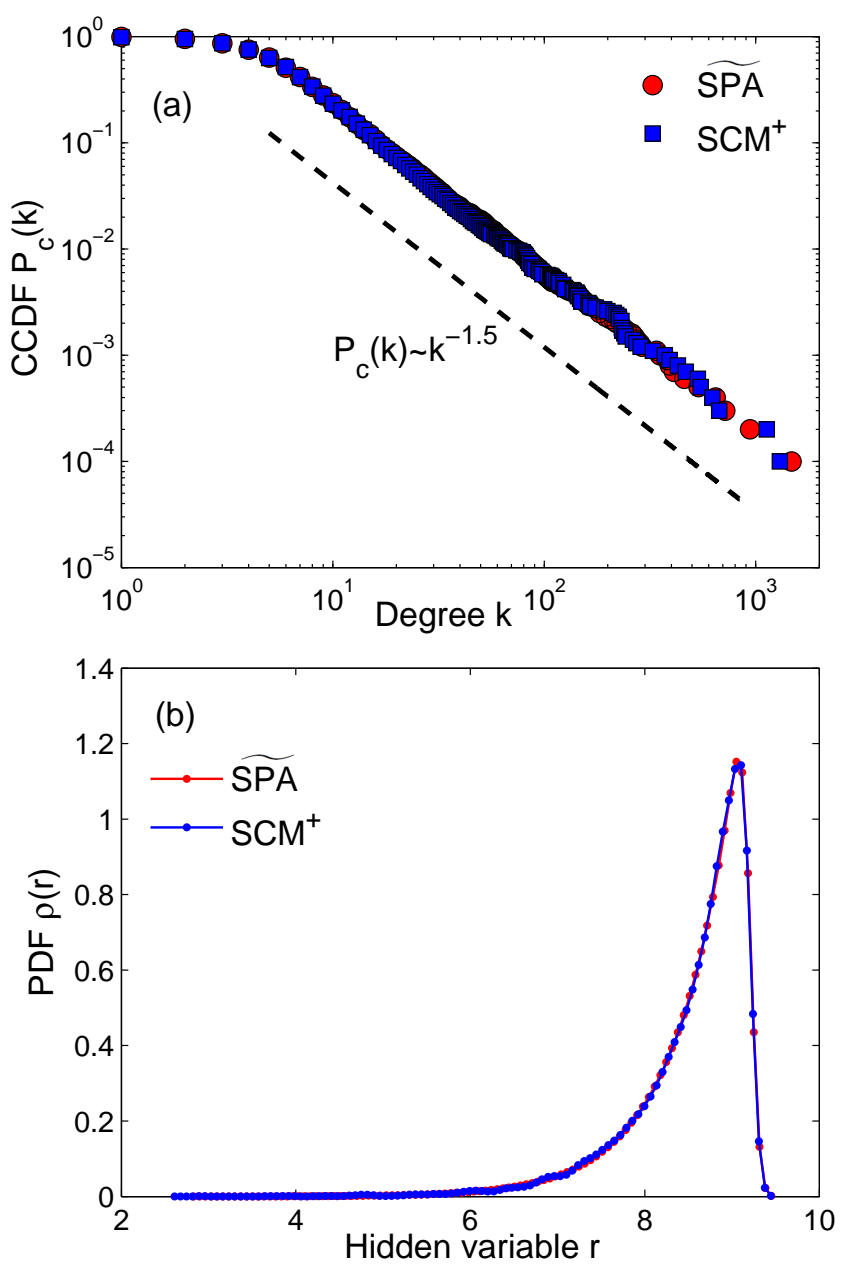

FIG. 2: $\widetilde{\text { SPA }}$ and $\mathbf{S C M}^{+}$networks. Panel (a) shows the empirical CCDF $P_{c}(k)=\sum_{k^{\prime}>k} \mathbb{P}\left(k^{\prime}\right)$ for two networks of size $N=10^{4}$ generated by $\widetilde{\mathrm{SPA}}$ and $\mathrm{SCM}^{+}$with $\bar{k}=10$ and $\gamma=2.5$, and the corresponding power-law fit. As expected, for both networks $P_{c}(k) \sim k^{-\gamma+1}$. Panel (b) shows the perfect match between the PDFs of the hidden variables in these two networks, illustrating the theoretical result $\rho_{\widetilde{\mathrm{SPA}}}(r)=$ $\rho_{\mathrm{SCM}}+(r)$.

Since $r$ and $r$ are the values of hidden variables of nodes $j$ and $i$ at time $N$, we have

$$
\begin{aligned}
& r=r_{j}(N)=\beta r_{j}+(1-\beta) \ln N \\
& r=r_{i}(N)=\beta r_{i}+(1-\beta) \ln N
\end{aligned}
$$

Finding from these equations $r_{i}$ and $r_{j}$ and substituting them into 54, we obtain

$$
\int_{i}^{N} p_{i j}^{\mathrm{int}}(s) d s=\int_{i}^{N} \frac{d s}{1+\frac{e^{r+\dot{r}}}{m_{\mathrm{int}}(1-\beta)}\left(\frac{s}{N}\right)^{2(1-\beta)}} .
$$

To proceed with analytic approximation, we need to use the classical limit for the Fermi-Dirac distribution, i.e. to drop the term 1 in the denominator. We have already used this approximation in 45 and 49 . In those cases, 
the second terms in the denominator were fully deterministic, and it was readily verifiable that they are much larger than 1, and therefore the approximations hold. In (57) however, both $r$ and $\dot{r}$ are random, $r, r \sim \rho_{\mathrm{SCM}^{+}}$, and a certain caution is required.

In what follows, we show that the expected value of $e^{r}$ in $\mathrm{SCM}^{+}$scales as $N$, and, therefore, $\frac{e^{r+\tilde{r}}}{N^{2(1-\beta)}} \propto N^{2 \beta} \geq$ $N \gg 1$. Indeed,

$$
\begin{aligned}
\left\langle e^{r}\right\rangle & =\int_{0}^{R_{\mathrm{SCM}}} e^{x+\sigma} \rho_{\mathrm{SCM}}(x) d x \\
& \approx \frac{e^{\sigma}}{\beta} \int_{0}^{R_{\mathrm{SCM}}} e^{x} e^{\frac{x-R_{\mathrm{SCM}}}{\beta}} d x \\
& =\frac{\bar{k}(1-\beta)^{2}}{\beta e^{\frac{R_{\mathrm{SCM}}}{\beta}}} \int_{0}^{R_{\mathrm{SCM}}} e^{\left(1+\frac{1}{\beta}\right) x} d x \\
& =\frac{\bar{k}(1-\beta)^{2}}{(1+\beta) e^{\frac{R_{\mathrm{SCM}}}{\beta}}\left(e^{\left(1+\frac{1}{\beta}\right) R_{\mathrm{SCM}}}-1\right)} \\
& \approx \frac{\bar{k}(1-\beta)^{2}}{1+\beta} e^{R_{S C M}}=\frac{N}{1+\beta} \propto N .
\end{aligned}
$$

Thus,

$$
\begin{gathered}
\int_{i}^{N} p_{i j}^{\mathrm{int}}(s) d s \approx \frac{(1-\beta) m_{\mathrm{int}}}{e^{r+\hat{r}} N^{-2(1-\beta)}} \int_{i}^{N} s^{-2(1-\beta)} d s \\
=\frac{(1-\beta) m_{\mathrm{int}}}{(2 \beta-1) e^{r+\hat{r}} N^{-2(1-\beta)}}\left(N^{2 \beta-1}-i^{2 \beta-1}\right) \\
=\frac{\bar{k}(1-\beta)^{2} N}{e^{r+\hat{r}}}-\frac{\bar{k}(1-\beta)^{2} N^{2(1-\beta)} i^{2 \beta-1}}{e^{r+\hat{r}}} .
\end{gathered}
$$

The probability of the external link is

$$
\begin{aligned}
p_{i j}^{\mathrm{ext}} & =\frac{1}{1+e^{r_{j}(i)+r_{i}-R_{i}^{\mathrm{ext}}}} \\
& \approx \frac{1}{1+e^{\beta r_{j}+(1-\beta) r_{i}-\ln m_{\mathrm{ext}}(1-\beta)}} \\
& =\frac{1}{1+\frac{e^{r+\frac{1-\beta}{\beta}}}{m_{\mathrm{ext}}(1-\beta) N^{\frac{1-\beta}{\beta}}}} \approx \frac{\bar{k}(1-\beta)^{2} N^{\frac{1-\beta}{\beta}}}{e^{r+\frac{1-\beta}{\beta} \dot{r}}},
\end{aligned}
$$

where the last approximation holds because, using (58), $e^{r+\frac{1-\beta}{\beta} \dot{r}} / N^{\frac{1-\beta}{\beta}} \propto N$. Combining $53, \sqrt{59}$ and 60 , we obtain that the probability that nodes $i$ and $j$ in SPA are connected is

$$
\begin{aligned}
& p_{\widetilde{\mathrm{SPA}}}(i, j)=\frac{\bar{k}(1-\beta)^{2} N}{e^{r+\dot{r}}} \\
& \quad-\frac{\bar{k}(1-\beta)^{2} N^{2(1-\beta)} i^{2 \beta-1}}{e^{r+\dot{r}}}+\frac{\bar{k}(1-\beta)^{2} N^{\frac{1-\beta}{\beta}}}{e^{r+\frac{1-\beta}{\beta} \dot{r}}} .
\end{aligned}
$$

To compare this probability with the connection probability in $\mathrm{SCM}^{+}$, we need to rewrite it fully in terms of hidden variables $r$ and $\dot{r}$. From $56, r_{i}=\frac{\tilde{r}}{\beta}-\frac{1-\beta}{\beta} \ln N$. Therefore,

$$
i=e^{\frac{\dot{r}}{\beta}-\frac{1-\beta}{\beta} \ln N}=e^{\frac{\dot{r}}{\beta}} N^{-\frac{1-\beta}{\beta}} .
$$

Substituting this expression into [61, we obtain after some algebra that the last two terms cancel out, and

$$
p_{\widetilde{\mathrm{SPA}}}(i, j)=\frac{\bar{k}(1-\beta)^{2} N}{e^{r+\hat{r}}} .
$$

It remains to show that 63 is, in fact, the connection probability in $\mathrm{SCM}^{+}$. Indeed,

$$
\begin{aligned}
p_{\mathrm{SCM}^{+}}(r, \dot{r}) & =\frac{1}{1+e^{r+\hat{r}-R_{\mathrm{SCM}^{+}}}} \\
= & \frac{1}{1+e^{r+\hat{r}-\ln N \bar{k}(1-\beta)^{2}}} \\
& =\frac{1}{1+\frac{e^{r+\dot{r}}}{N k(1-\beta)^{2}}} \approx \frac{\bar{k}(1-\beta)^{2} N}{e^{r+\hat{r}}},
\end{aligned}
$$

where the last approximation holds because $\frac{e^{r+r}}{N} \propto N$. In Appendix A.2, we show the high accuracy of this approximation with simulation. Fig. 3(a) juxtaposes the empirical connection probabilities in SPA and $\mathrm{SCM}^{+}$networks, and the corresponding approximation in (64). Recall that the match between the connection probabilities is the second condition (16) for two ensembles of random graphs with hidden variables to be equivalent.

Thus, we proved that, for large networks, $\widetilde{\mathrm{SPA}}$ is equivalent to $\mathrm{SCM}^{+}$in the strong sense. That is, for any $N \gg 1, \widetilde{\mathrm{SPA}}$ and $\mathrm{SCM}^{+}$generate graphs $G \in \mathcal{G}_{N}$ with the same probability, $\mathbb{P}_{\widetilde{\mathrm{SPA}}}(G)=\mathbb{P}_{\mathrm{SCM}^{+}}(G)$. Therefore, the expected values of all graph properties in the ensemble, not only of the degree distributions in Fig. 2(a), are the same. As an example, Fig. 3(b) shows the vertex-tovertex distance distribution $d(l)$, which is the distribution of hop lengths $l$ of shortest paths between nodes in the network, or the probability that a random pair of nodes are at the distance of $l$ hops from each other.

The following diagram summarizes the relationships between the four considered network models

$$
\mathrm{SPA} \rightsquigarrow \widetilde{\mathrm{SPA}} \stackrel{s}{\approx} \mathrm{SCM}^{+} \stackrel{s}{=} \mathrm{SCM}
$$

where $\stackrel{s}{=}$ denotes the strong model equivalence, $\stackrel{s}{\approx}$ is an approximate strong equivalence that becomes exact in the sparse graph limit $(N \rightarrow \infty, \bar{k} \ll N)$, and $\rightsquigarrow$ denotes the model transformation allowing internal links. Furthermore,

$$
\left.\left.\mathrm{SPA}\right|_{\beta=1 / 2} \stackrel{s}{=} \widetilde{\mathrm{SPA}}\right|_{\beta=1 / 2},
$$

that is, SPA and $\widetilde{\mathrm{SPA}}$ are strongly equivalent if $\beta=1 / 2$, i.e., $\gamma=3$. 

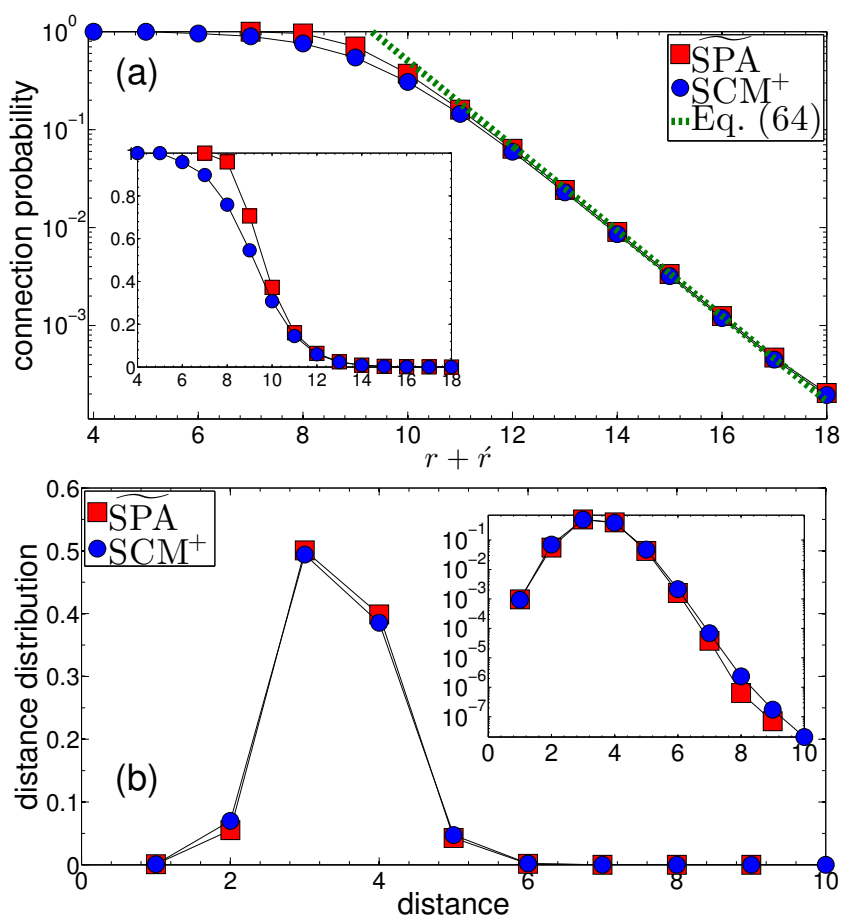

FIG. 3: Connection probabilities and vertex-to-vertex distance distribution in $\widetilde{\mathrm{SPA}}$ and $\mathrm{SCM}^{+}$networks. Panel (a) shows the empirical connection probabilities in networks of size $N=10^{4}$ generated by $\widetilde{\mathrm{SPA}}$ and $\mathrm{SCM}^{+}$with $\bar{k}=10$ and $\gamma=2.5$. The $y$-axis in the main plot is in logarithmic scale. As expected, the connection probabilities match remarkably well. The plot also shows the corresponding approximation given in 64). As expected, this approximation holds very well for sufficiently large values of $r+\dot{r}$, which correspond to the vast majority of node pairs, cf. Fig. 2(b) and Appendix A.2 Panel (b) shows the distance distributions in the same networks. The $y$-axis in the inset is in logarithmic scale. As a consequence of the equivalence of $\widehat{\mathrm{SPA}}$ and $\mathrm{SCM}^{+}$, the distance distributions also match remarkably well, as expected. In all cases the results were averaged across 100 networks.

\section{E. Hamiltonians of $\mathrm{SCM}^{+}, \widetilde{\mathrm{SPA}}$, and SPA}

As discussed in Section VA, SCM is the ERGM model with Hamiltonian

$$
\begin{aligned}
H_{\mathrm{SCM}} & =\sum_{i=1}^{N} k_{i} r_{i}-M R_{\mathrm{SCM}} \\
& =\sum_{i}^{N} k_{i} r_{i}-\frac{(\ln N-\sigma)}{2} \sum_{i=1}^{N} k_{i} .
\end{aligned}
$$

Since $\mathrm{SCM}^{+}$is strongly equivalent to $\mathrm{SCM}$ - the two models differ only by parametrization of hidden variables
- $\mathrm{SCM}^{+}$must have the same Hamiltonian. Indeed,

$$
\begin{aligned}
H_{\mathrm{SCM}^{+}} & =\sum_{i=1}^{N} k_{i} r_{i}^{+}-M R_{\mathrm{SCM}^{+}} \\
& =\sum_{i=1}^{N} k_{i}\left(r_{i}+\sigma\right)-\frac{(\ln N+\sigma)}{2} \sum_{i=1}^{N} k_{i} \\
& =\sum_{i=1}^{N} k_{i} r_{i}-\frac{(\ln N-\sigma)}{2} \sum_{i=1}^{N} k_{i}=H_{\mathrm{SCM}} .
\end{aligned}
$$

Further, since $\widetilde{\mathrm{SPA}}$ is strongly equivalent to $\mathrm{SCM}^{+}$and the distributions of hidden variables in these two models are the same, the ERGM Hamiltonian of SPA is

$$
\begin{aligned}
H_{\widetilde{\mathrm{SPA}}} & =\sum_{i=1}^{N} k_{i} r_{i}-M R_{\mathrm{SCM}^{+}} \\
& =\sum_{i=1}^{N} k_{i} r_{i}-\frac{(\ln N+\sigma)}{2} \sum_{i=1}^{N} k_{i} \\
& =\sum_{i=1}^{N} k_{i} r_{i}-\frac{\ln \left(N \bar{k}(1-\beta)^{2}\right)}{2} \sum_{i=1}^{N} k_{i} .
\end{aligned}
$$

Finally, since $\widetilde{\mathrm{SPA}}$ becomes manifestly identical to SPA as $\beta \rightarrow 1 / 2$, or, equivalently, as $\gamma \rightarrow 3$, we can write the ERGM Hamiltonian for SPA

$$
H_{\mathrm{SPA}}=\sum_{i=1}^{N} k_{i} r_{i}-\frac{\ln (N \bar{k} / 4)}{2} \sum_{i=1}^{N} k_{i} .
$$

This result means that if $\beta=1 / 2(\gamma=3)$, then, in the sparse graph limit, SPA is exactly ERGM with Hamiltonian 70p. We note that this case corresponds to the original Barabási-Albert model with the scaling exponent $\gamma=3[12$. If $\beta \neq 1 / 2$, then $\sqrt{70}$ is an approximate Hamiltonian of SPA. To the best of our knowledge, this is the first result where the preferential attachment model is represented as an exponential random graph model with explicitly written Hamiltonian. Even more remarkably, as we show in the next section, a Hamiltonian that is very similar to the ERGM Hamiltonian (70) describes the Hamiltonian dynamics of growing networks in SPA.

\section{HAMILTONIAN DYNAMICS OF SPA}

The key idea in deriving the ERGM Hamiltonian of SPA 70 was to construct a modified model $\widetilde{\mathrm{SPA}}$ that: a) is strongly equivalent to a graph ensemble with a known Hamiltonian; and b) coincides with SPA under certain values of the model parameters. Here we adopt a similar strategy: we study the Hamiltonian dynamics of growing $\widetilde{\mathrm{SPA}}-$ networks, and the corresponding results for the Hamiltonian dynamics of SPA are obtained as a special case with $\beta=1 / 2$. 
The ERGM Hamiltonian of $\widetilde{\mathrm{SPA}} 69$ suggests that the canonical coordinates $\left\{q_{i}, p_{i}\right\}$ of a growing network are the node degrees $k_{i}$ and hidden variables $r_{i}$. An immediate technical problem we face, however, is that both node degrees $k_{i}$ and network time $i$ are discrete. We overcome this obstruction as follows. First, inspired by the mapping between the hyperbolic and de Sitter spaces in [4], we define

$$
t=\beta \ln i,
$$

and treat $t$ as a continuous time. The geometric duality between de Sitter spacetime, which is asymptotically the spacetime of our accelerating universe, and hyperbolic space, which is a latent space underlying real complex networks 42, 43, allows to interpret $t$ as the rescaled cosmological time. Second, instead of the exact (discrete) degree $k_{i}(t)$ of node $i$, born at time $t_{i}=\beta \ln i$, at a later time $t>t_{i}$, we use its expected degree $\kappa_{i}(t)$, which is a continuous function of $t$, for $t>t_{i}$.

Our next goal is to derive the time evolution of the canonical coordinates $\left\{\kappa_{i}(t), r_{i}(t)\right\}$ in the growing SPAand $\widetilde{\mathrm{SPA}}-$ networks. Given that in network time $i, r_{j}(i)=$ $\beta r_{j}+(1-\beta) r_{i}$, in the rescaled cosmological time the evolution of the hidden variable $r_{i}$ of node $i$ - in both SPA and $\widetilde{\mathrm{SPA}}$ - is

$$
r_{i}(t)=t_{i}+\frac{1-\beta}{\beta} t
$$

where $t_{i}=\beta \ln i$ is the birth time of node $i$. The expected node degrees, however, evolve differently in SPA and $\widetilde{\mathrm{SPA}}$, as we show below.

\section{A. Evolution of the expected node degree in SPA} is

The expected degree of node $j$ at network time $i>j$

$$
\begin{aligned}
\kappa_{j}(i) & =\sum_{s<j} p_{j s}+\sum_{s>j}^{i} p_{s j} \\
& \approx \int_{0}^{j} \frac{d s}{1+e^{r_{s}(j)+r_{j}-R_{j}}}+\int_{j}^{i} \frac{d s}{1+e^{r_{j}(s)+r_{s}-R_{s}}} .
\end{aligned}
$$

The first term is the expected degree of node $j$ upon its birth, and the integral is calculated in the same way as the integral in (45), and it equals to $m$. The second term is

$$
\begin{aligned}
& \int_{j}^{i} \frac{d s}{1+e^{r_{j}(s)+r_{s}-R_{s}}} \approx \int_{j}^{i} \frac{d s}{1+\frac{j^{\beta} s^{1-\beta}}{m(1-\beta)}} \\
& \approx \frac{m(1-\beta)}{j^{\beta}} \int_{j}^{i} s^{\beta-1} d s=\frac{m(1-\beta)}{\beta}\left(\left(\frac{i}{j}\right)^{\beta}-1\right),
\end{aligned}
$$

and, therefore,

$$
\kappa_{j}(i)=\frac{m(1-\beta)}{\beta}\left(\frac{i}{j}\right)^{\beta}+\frac{m(2 \beta-1)}{\beta} .
$$

This expression coincides exactly with the expression obtained in 14 for the expected degree of a node in the sharp preferential attachment model, where the link attraction probability is a liner function of the node's exact degree (versus expected degree).

Finally, in the rescaled cosmological time,

$$
\kappa_{i}(t)=\frac{m(1-\beta)}{\beta} e^{t-t_{i}}+\frac{m(2 \beta-1)}{\beta} .
$$

\section{B. Evolution of the expected node degree in SPA}

Similarly, the expected degree of node $j$ at network time $i>j$ is

$$
\kappa_{j}(i)=\sum_{s<j} p_{j s}^{\mathrm{ext}}+\sum_{s>j}^{i} p_{s j}^{\mathrm{ext}}+\sum_{s>j}^{i} \sum_{\substack{a<s \\ a \neq j}} p_{a j}^{\mathrm{int}}(s) .
$$

The first two terms are exactly the same as in SPA 73 with $m$ replaced by $m_{\text {ext }}$. The last term is the expected contribution to the $j$ 's degree from internal links,

$$
\begin{aligned}
& \sum_{s>j}^{i} \sum_{\substack{a<s \\
a \neq j}} p_{a j}^{\mathrm{int}}(s) \approx \int_{j}^{i} \int_{0}^{s} \frac{d a d s}{1+e^{r_{a}(s)+r_{j}(s)-R^{\mathrm{int}}}} \\
& =\int_{j}^{i} \int_{0}^{s} \frac{d a d s}{1+\frac{a^{\beta} j^{\beta} s^{2(1-\beta)}}{m_{\mathrm{int}}(1-\beta)}} \\
& \approx \frac{m_{\mathrm{int}}(1-\beta)}{j^{\beta}} \int_{j}^{i} \int_{0}^{s} a^{-\beta} s^{-2(1-\beta)} d a d s \\
& =\frac{m_{\mathrm{int}}}{\beta}\left(\left(\frac{i}{j}\right)^{\beta}-1\right) .
\end{aligned}
$$

Combining all terms together, we obtain

$$
\kappa_{j}(i)=m_{\mathrm{ext}}\left(\frac{i}{j}\right)^{\beta}
$$

and, in the rescaled cosmological time,

$$
\kappa_{i}(t)=m_{\mathrm{ext}} e^{t-t_{i}}=\bar{k}(1-\beta) e^{t-t_{i}} .
$$

If $\beta=1 / 2$, then, as expected, the expressions for the expected node degrees in SPA and $\widetilde{\mathrm{SPA}}$ become identical.

\section{Dynamic Hamiltonians}

We now have all the ingredients necessary to derive the Hamiltonian describing the dynamics of network growth

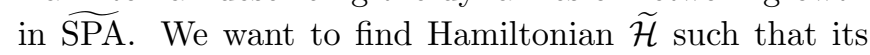
Hamilton's equations have 72 and 80 as the solutions.

Let $\widetilde{\mathcal{H}}_{i}\left(\kappa_{i}, r_{i}, t\right)$ be the energy contribution of node $i$ at time $t$ to the total network Hamiltonian

$$
\widetilde{\mathcal{H}}=\sum_{i=1}^{N} \widetilde{\mathcal{H}}_{i}\left(\kappa_{i}, r_{i}, t\right)
$$


Then Hamilton's equations for node $i$ are

$$
\dot{\kappa}_{i}=\frac{\partial \widetilde{\mathcal{H}}_{i}}{\partial r_{i}} \quad \text { and } \quad \dot{r}_{i}=-\frac{\partial \widetilde{\mathcal{H}}_{i}}{\partial \kappa_{i}} .
$$

Formally integrating these equations, and noting that

$$
\dot{\kappa}_{i}=m_{\mathrm{ext}} e^{t-t_{i}} \quad \text { and } \quad \dot{r}_{i}=\frac{1-\beta}{\beta},
$$

we can write the solution in the following form

$$
\widetilde{\mathcal{H}}_{i}\left(\kappa_{i}, r_{i}, t\right)=m_{\text {ext }} e^{t-t_{i}} r_{i}-\frac{1-\beta}{\beta} \kappa_{i}+\xi_{i}(t),
$$

where $\xi_{i}(t)$ is some function of time $t$ and model parameters $\bar{k}$ and $\beta$.

Since $\xi_{i}(t)$ does not affect the equations of motion 82 , in principle, it can be chosen arbitrary. Remarkably, $\xi_{i}(t)$ can be chosen in such way that it is the same for all nodes, and the resulting total Hamiltonian $\widetilde{\mathcal{H}}$ is identical to the ERGM Hamiltonian $H_{\widetilde{\mathrm{SPA}}}$ with node degrees replaced by their expected values. Indeed, let

$$
\xi_{i}(t)=\frac{\bar{k}}{2}\left(\frac{2(1-\beta)}{\beta}-\sigma-\frac{t}{\beta}\right) .
$$

Now, consider a network snapshot at time

$$
T=\beta \ln N \gg 1,
$$

with given (current) values of $\kappa_{i}$ and $r_{i}$. Using 80, 85, and (86), we can rewrite the Hamiltonian of node $i$ as follows

$$
\widetilde{\mathcal{H}}_{i}=\kappa_{i} r_{i}-\frac{1-\beta}{\beta}\left(\kappa_{i}-\bar{k}\right)-\frac{\bar{k}(\ln N+\sigma)}{2} .
$$

The total Hamiltonian of the snapshot is then

$$
\widetilde{\mathcal{H}}=\sum_{i=1}^{N} \kappa_{i} r_{i}-\frac{1-\beta}{\beta}\left(\sum_{i=1}^{N} \kappa_{i}-N \bar{k}\right)-\frac{N \bar{k}(\ln N+\sigma)}{2} .
$$

Since $\sum_{i=1}^{N} \kappa_{i}=N \bar{k}$, we obtain that

$$
\begin{aligned}
\widetilde{\mathcal{H}} & =\sum_{i=1}^{N} \kappa_{i} r_{i}-\frac{(\ln N+\sigma)}{2} \sum_{i=1}^{N} \kappa_{i} \\
& =\sum_{i=1}^{N} \kappa_{i} r_{i}-\frac{\ln \left(N \bar{k}(1-\beta)^{2}\right)}{2} \sum_{i=1}^{N} \kappa_{i},
\end{aligned}
$$

which is exactly the expected ERGM Hamiltonian (69). Note that since the ERGM Hamiltonian can be interpreted as the energy of a given network snapshot at some time $t$, the dynamic Hamiltonian with $\xi_{i}(t)$ in 85 yields indeed the expected energy of the snapshot.

To obtain the dynamic SPA Hamiltonian, all we need to do is to set $\beta=1 / 2$ in the above derivations. The energy contribution of node $i$ is then

$$
\mathcal{H}_{i}=\frac{\bar{k}}{2} e^{t-t_{i}}-\kappa_{i}+\bar{k}\left(1-\frac{1}{2} \ln \frac{\bar{k}}{4}-t\right),
$$

and the total Hamiltonian that describes the dynamics of growing networks in SPA is

$$
\mathcal{H}=\sum_{i=1}^{N} \kappa_{i} r_{i}-\frac{\ln (N \bar{k} / 4)}{2} \sum_{i=1}^{N} \kappa_{i} .
$$

As expected, this Hamiltonian is exactly the ERGM Hamiltonian of SPA 70 with the node degrees replaced by their expected values.

\section{CONCLUSION}

We have studied the dynamics of networks growing according to preferential attachment, and obtained two important results. First, we have shown that soft preferential attachment can be casted as an equilibrium exponential random graph model, nearly identical to the soft configuration model. In other words, the ensemble of random graphs that preferential attachment generates is nearly identical to the equilibrium ensemble of random graphs with power-law degree distributions, meaning that preferential attachment and configuration model generate any graph $G$ of any size $N$ with the same probability $\mathbb{P}(G)$. In general, this result is important because equilibrium network models tend to be more amenable for analytic treatment. In particular, this result, for the first time to the best of our knowledge, provides an explicit expression $\mathbb{P}(G) \propto \exp [-H(G)]$ with Hamiltonian $H(G)$ for the probability $\mathbb{P}(G)$ that preferential attachment generates any given network $G$. The knowledge of $\mathbb{P}(G)$ can be used, for example, for answering the question of how likely it is that a given real network has been grown according to preferential attachment. This question can now be answered by standard techniques, such as comparing the probabilities $\mathbb{P}(G)$ of the typical preferential attachment networks and the real network under study. Another application is an alternative simpler method to generate preferential attachment networks, which has already been implemented and publicly released as a part of a more general software package that generates random hyperbolic graphs 48.

Second, we have demonstrated that the growing dynamics of preferential attachment networks is Hamiltonian. Remarkably, the Hamiltonian $\mathcal{H}$ (89) that defines the equations of motion (72 80 describing network dynamics is nearly identical to the ERG Hamiltonian $H$ 69. The only difference between the two is that the exact node degrees in $H$ are replaced by their expected values in $\mathcal{H}$.

These results may appear quite surprising at the first glance, but there is an intuitive explanation. On the one hand, the equilibrium Hamiltonian $H(G)$ in the soft configuration model is the energy of graph $G$ in the Boltzmann distribution $\mathbb{P}(G)$ of this exponential random graph model. This energy is the sum of energies of all edges in graph $G$, and one can check that the energy of edge $\{i j\}$ is simply the sum of $i$ 's and $j$ 's Lagrange multipliers 
$r_{i}+r_{j}$. On the other hand, as shown in Section $\mathrm{V}$, soft preferential attachment, at each time $t$, is also a similar exponential random graph model, with hidden variables $r_{i}$ playing the role of Lagrange multipliers, and the energy of edge $\{i j\}$ at time $t$ is also $r_{i}(t)+r_{j}(t)$.

In simpler terms, the reason behind this equivalence is quite physical: both the dynamic Hamiltonian in preferential attachment and the equilibrium Hamiltonian in the configuration model are system energies, albeit the established equivalence between the growing and equilibrium representations of the same system is slightly atypical in physics 28.

Very few real networks can be adequately modeled as random graphs in the configuration model, which suggests that some additional terms must be added to the Hamiltonian to adequately describe the dynamics of different real networks.In this context, it is an interesting observation that the ERG ensemble that we found to be equivalent to preferential attachment is a degenerate case of the more general geometric network ensembles, which can be considered as a Fermi gas in a hyperbolic space, whose symmetry group is the Lorentz group [42,43]. This observation calls for extending the developed canonical formalism for network analysis to this more general geometric case with non-degenerate symmetries. This extension is a highly non-trivial task for a number of technical reasons, but if successful, it may shed some light on the second question we raised in the introduction, concerning small-scale dynamics of networks.

We have shown that preferential attachment can be formulated within the canonical formalism, in which the time evolution of a system is described by Hamilton's equations $\dot{q}=\partial \mathcal{H} / \partial p$ and $\dot{p}=-\partial \mathcal{H} / \partial q$. The traditional application of the Hamiltonian formalism in mathematical physics [49] deals with the following direct problem: given a Hamiltonian $\mathcal{H}$, which in most cases is the energy of the system, find the solution of the corresponding dynamical equations of motion. However, in physics history, the problem has almost always been inverse: first, chronologically, the equations of motion are found by some other, usually experimental methods, and only much later it is recognized by theoreticians that these equations are solutions of some Hamiltonian or Lagrangian systems defined by their symmetry groups. This was the case in most physics theories, from classical mechanics [49] to general relativity [20]. Our understanding of network dynamics seems to have been driven along a similar historic path. First preferential attachment was suggested as a likely mechanism responsible for the emergence of scale-free degree distributions 12 14, experimentally validated for many real networks $15-18$. And only fifteen years later have we recognized that the preferential attachment dynamics 72 80 is Hamiltonian 89.

We emphasize however that these results hold only for the soft versions of preferential attachment and configuration model. The difference between the soft configuration model with a fixed expected scale-free degree sequence and the configuration model in which the ex- pected degree sequence is sampled for each graph from a fixed scale-free distribution has been recently quantified in [50. This difference is well-behaved, in the sense that the entropy distribution in the latter ensemble is self-averaging, meaning that its relative variance vanishes in the thermodynamic limit. However, it is known that the soft (canonical) and sharp (microcanonical) configuration models are different even in the thermodynamic limit - the ensemble distributions do not converge in the limit [31, 51. To the best of our knowledge, there are no results of this sort concerning the difference between the soft and sharp versions of preferential attachment, but one could expect them to be different as well. Therefore the existence of any connections between sharp configuration model and sharp preferential attachment, and the possibility to formulate the latter within the canonical approach, remain to be open questions.

\section{Acknowledgments}

We thank Paul Krapivsky for useful discussions. This work was supported by DARPA grant No. HR0011-121-0012; NSF grants No. CNS-1344289, CNS-1442999, CNS-0964236, CNS-1441828, CNS-1039646, and CNS1345286; by Cisco Systems; and by a Marie Curie International Reintegration Grant within the 7th European Community Framework Programme.

\section{Appendix}

\section{A.1. SPA, $\widetilde{\mathbf{S P A}}$, and $\mathbf{S C M}^{+}$as $\beta \rightarrow 1(\gamma \rightarrow 2)$}

All three models $-\mathrm{SPA}, \widetilde{\mathrm{SPA}}$, and $\mathrm{SCM}^{+}$- have singularities at $\beta=1$. In this Appendix, we investigate to what models they degenerate in the limit $\beta \rightarrow 1$.

The SPA model has a well-defined limit. Indeed, since

$$
\begin{aligned}
& R_{i} \rightarrow r_{i}-\ln \frac{r_{i}}{m} \text { and } \\
& p_{i j} \rightarrow \frac{1}{1+e^{r_{j}+\ln r_{i}-\ln m}}=\frac{1}{1+\frac{j \ln i}{m}},
\end{aligned}
$$

as $\beta \rightarrow 1$, SPA converges to the following simple model. To generate a network of size $N$ with average degree $\bar{k}$ and power-law exponent $\gamma=2$, for each new node $i=$ $1, \ldots, N$, connect node $i$ to each existing node $j<i$ with probability

$$
p_{i j}=\frac{1}{1+\frac{j \ln i}{m}}, \quad m=\frac{\bar{k}}{2} .
$$

In what follows, we show that the average degree in large networks generated by this limiting model is indeed $\bar{k}$. At time $N$, the expected degree of node $i$ is

$$
\kappa_{i}(N)=\sum_{j<i} p_{i j}+\sum_{j>i}^{N} p_{j i} .
$$


The first sum is the expected contribution to the $i$ 's degree from older nodes,

$$
\sum_{j<i} p_{i j} \approx \frac{m}{\ln i} \sum_{j=1}^{i} \frac{1}{j}=\frac{m}{\ln i} H_{i} \approx m,
$$

where $H_{i}=\sum_{j=1}^{i} \frac{1}{j} \approx \ln i$ is the $i^{\text {th }}$ harmonic number. The second sum in A.95 is the expected contribution to the degree of node $i$ from younger nodes,

$$
\sum_{j>i}^{N} p_{j i} \approx \frac{m}{i} \int_{i}^{N} \frac{d j}{\ln j}=\frac{m}{i}(\operatorname{li}(N)-\operatorname{li}(i)),
$$

where $\operatorname{li}(x)$ is the logarithmic integral function. Therefore,

$$
\kappa_{i}(N)=m+\frac{m}{i}(\operatorname{li}(N)-\operatorname{li}(i)) .
$$

The expected average degree in the network at time $N$ is then

$$
\begin{aligned}
\kappa(N) & =\frac{1}{N} \sum_{i=1}^{N} \kappa_{i}(N) \\
& =m+\frac{m \operatorname{li}(N) H_{N}}{N}-\frac{m}{N} \sum_{i<N} \frac{\operatorname{li}(i)}{i} .
\end{aligned}
$$

Since $\operatorname{li}(N) \approx \frac{N}{\ln N}$ and $H_{N} \approx \ln N$, the second term is approximately $m$. The last term can be approximated as follows

$$
\sum_{i<N} \frac{\operatorname{li}(i)}{i} \approx \int_{0}^{N} \frac{\operatorname{li}(i) d i}{i}=\operatorname{li}(N) \ln N-N .
$$

Therefore, we finally have

$$
\begin{aligned}
\kappa(N) & =2 m-\frac{m}{N}(\operatorname{li}(N) \ln N-N) \\
& =3 m-\frac{m \operatorname{li}(N) \ln N}{N} \approx 2 m=\bar{k} .
\end{aligned}
$$

Figure 4 illustrates how this approximation becomes more accurate as the network size increases.

The SPA model completely degenerates as $\beta \rightarrow 1$. Namely, $R_{i}^{\text {ext }} \rightarrow-\infty$ and $R^{\text {int }} \rightarrow-\infty$, and, therefore, $p_{i j}^{\text {ext }} \rightarrow 0$ and $p_{a b}^{\text {int }}(i) \rightarrow 0$. This means that the limiting model generates networks with no links. Remarkably, even in the limit $\beta \rightarrow 1, \widetilde{\mathrm{SPA}}$ remains strongly equivalent to $\mathrm{SCM}^{+}$. To prove this, we need to show that in this limit $\mathrm{SCM}^{+}$also generates networks without links.

The connection probability in $\mathrm{SCM}^{+}$is

$$
p_{i j}=\frac{1}{1+e^{r_{i}^{+}+r_{j}^{+}-R_{\mathrm{SCM}^{+}}}},
$$

where $r_{i}^{+}=r_{i}+\ln \left(\bar{k}(1-\beta)^{2}\right), r_{i} \sim \rho_{\mathrm{SCM}}(r)$, and $R_{\mathrm{SCM}^{+}}=\ln \left(N \bar{k}(1-\beta)^{2}\right)$. Since

$$
e^{r_{i}^{+}+r_{j}^{+}-R_{\mathrm{SCM}^{+}}}=\frac{e^{r_{i}} e^{r_{j}}}{N} \bar{k}(1-\beta)^{2},
$$

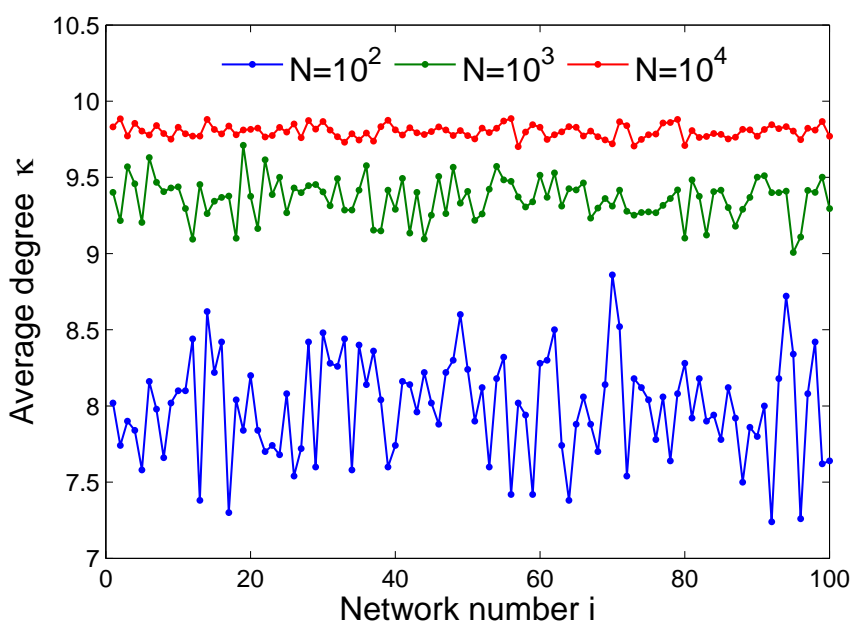

FIG. 4: Average degree in SPA networks with $\beta=1$ $(\gamma=2)$. For $N=10^{2}, 10^{3}$, and $10^{4}$, the plot shows the fluctuation of the average degree in 100 independent networks generated by the limiting $(\beta=1)$ SPA model with $\bar{k}=10$. As expected, the lager the network size $N$, the more accurate the approximation A.101.

and the expected value

$$
\begin{aligned}
\left\langle e^{r_{i}}\right\rangle & =\int_{0}^{R_{\mathrm{SCM}}} e^{r} \rho_{\mathrm{SCM}}(r) d r \\
& \approx \int_{0}^{R_{\mathrm{SCM}}} e^{r} e^{r-R_{\mathrm{SCM}}} d r \\
& =\frac{e^{R_{\mathrm{SCM}}}-e^{-R_{\mathrm{SCM}}}}{2} \rightarrow \frac{N}{2 \bar{k}(1-\beta)^{2}}
\end{aligned}
$$

we have that

$$
e^{r_{i}^{+}+r_{j}^{+}-R_{\mathrm{SCM}^{+}}} \approx \frac{N}{4 \bar{k}(1-\beta)^{2}} \rightarrow \infty .
$$

This means that the connection probability in $\mathrm{SCM}^{+}$ converges to zero, $p_{i j} \rightarrow 0$, as $\beta \rightarrow 1$, and therefore, even in this degenerate regime $\widetilde{\mathrm{SPA}}$ and $\mathrm{SCM}^{+}$are strongly equivalent.

\section{A.2. Accuracy of the classical limit approximation for the Fermi-Dirac distribution in $\mathrm{SCM}^{+}$}

In Section VD, we used the classical limit for the Fermi-Dirac distribution in the $\mathrm{SCM}^{+}$model

$$
p_{\mathrm{SCM}^{+}}\left(r, r^{\prime}\right) \approx \hat{p}_{\mathrm{SCM}^{+}}(r, \dot{r})=\frac{\bar{k}(1-\beta)^{2} N}{e^{r+\dot{r}}} .
$$

Here we show with simulations that this approximation is very accurate in large networks. As an example, we consider networks with $\bar{k}=10$ and $\gamma=2.5$. First, we generate $N$ hidden variables $r$ from distribution $\rho_{\mathrm{SCM}^{+}}(r)$, and then, for each of the $\left(\begin{array}{c}N \\ 2\end{array}\right)$ pairs of nodes with hidden 


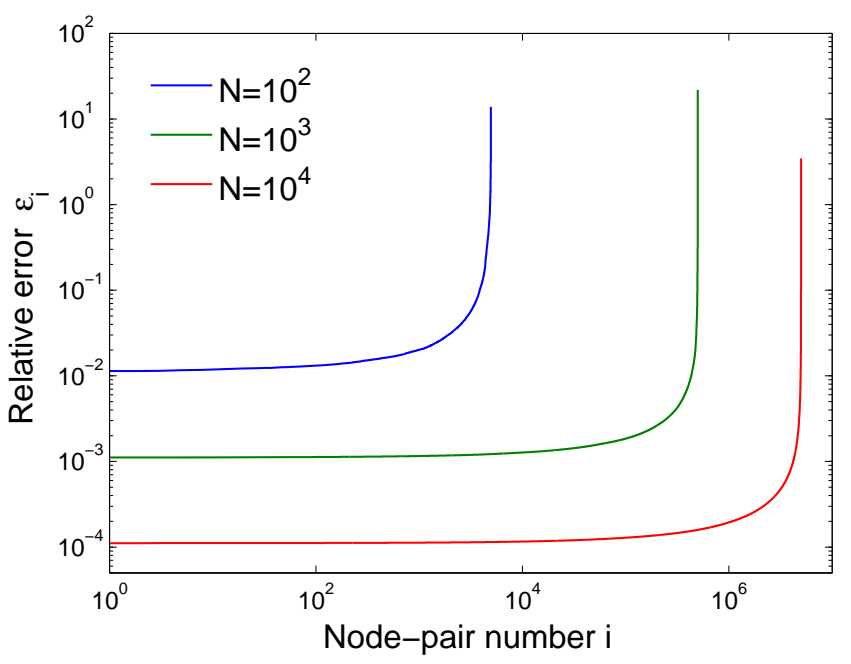

FIG. 5: Accuracy of the classical limit approximation. For $N=10^{2}, 10^{3}$, and $10^{4}$, the percentage of node-pairs with the relative error larger than $5 \%(1 \%)$ is, respectively, $40.5 \%$ $(100 \%), 1.8 \%(13.6 \%)$, and $0.08 \%$ (1\%). variables $r$ and $r$, we compute the relative error of the connection probability approximation A.106

$$
\varepsilon=\frac{\left|p_{\mathrm{SCM}^{+}}(r, \dot{r})-\hat{p}_{\mathrm{SCM}^{+}}\left(r, r^{\prime}\right)\right|}{p_{\mathrm{SCM}^{+}}(r, \dot{r})}
$$

Figure 5 shows the relative errors sorted in the increasing order for network sizes $N=10^{2}, 10^{3}$, and $10^{4}$. As expected, the larger the network, the smaller the classical limit approximation error.
[1] M. E. J. Newman, Networks: An Introduction (Oxford University Press, Oxford, 2010).

[2] D. Easley and J. Kleinberg, Networks, Crowds, and Markets: Reasoning about a Highly Connected World (Cambridge University Press, Cambridge, 2010).

[3] S. N. Dorogovtsev, Lectures on Complex Networks (Oxford University Press, Oxford, 2010).

[4] S. Meloni, A. Arenas, and Y. Moreno, Proc Natl Acad Sci USA 106, 16897 (2009).

[5] R. Pastor-Satorras and A. Vespignani, Phys. Rev. Lett. 86, 3200 (2001)

[6] W. Li, A. Bashan, S. V. Buldyrev, H. E. Stanley, and S. Havlin, Phys. Rev. Lett. 108, 228702 (2012).

[7] L. Daqing, J. Yinan, K. Rui, and S. Havlin, Scientific Reports 4 (2014).

[8] E. Kolaczyk, Statistical Analysis of Network Data (Springer, New York, 2009).

[9] A. Z. Jacobs and A. Clauset, in NIPS Workshop on Networks: From Graphs to Rich Data (2014).

[10] H. Owhadi, C. Scovel, and T. Sullivan, Electron. J. Statist. 9, 1 (2015).

[11] K. P. Burnham and D. R. Anderson, Model Selection and Multimodel Inference: A Practical InformationTheoretic Approach (Springer Science and Business Media, New York, NY, 2002).

[12] A.-L. Barabási and R. Albert, Science 286, 509 (1999).

[13] P. L. Krapivsky, S. Redner, and F. Leyvraz, Phys Rev Lett 85, 4629 (2000).

[14] S. N. Dorogovtsev, J. F. F. Mendes, and A. N. Samukhin, Phys Rev Lett 85, 4633 (2000).

[15] M. E. J. Newman, Phys. Rev. E 64, 025102 (2001).

[16] A. Barabási, H. Jeong, Z. Néda, E. Ravasz, A. Schubert, and T. Vicsek, Physica A 311, 590 (2002).

[17] A. Vázquez, R. Pastor-Satorras, and A. Vespignani, Phys Rev E 65, 066130 (2002).

[18] H. Jeong, Z. Néda, and A. L. Barabási, EPL (Europhysics
Letters) 61, 567 (2003).

[19] L. H. Ryder, Quantum Field Theory (Cambridge University Press, Cambridge, 1996).

[20] R. M. Wald, General Relativity (University of Chicago Press, Chicago, 2010).

[21] R. Solomonoff and A. Rapoport, Bull. Math. Biophys. 13, 107 (1951).

[22] P. Erdős and A. Rényi, Publ. Math. 6, 290 (1959).

[23] P. Erdős and A. Rényi, Publ. Math. Inst. Hung. Acad. Sci. 5, 17 (1960).

[24] S. N. Dorogovtsev, J. F. F. Mendes, and A. N. Samukhin, Nucl Phys B 666, 396 (2003).

[25] J. Berg and M. Lässig, Phys. Rev. Lett. 89, 228701 (2002).

[26] I. Farkas, I. Dernyi, G. Palla, and T. Vicsek, in Complex Networks, edited by E. Ben-Naim, H. Frauenfelder, and Z. Toroczkai (Springer Berlin Heidelberg, 2004), vol. 650 of Lecture Notes in Physics, pp. 163-187.

[27] M. Baiesi and S. S. Manna, Phys. Rev. E 68, 047103 (2003).

[28] D. Krioukov and M. Ostilli, Phys. Rev. E 88, 022808 (2013).

[29] J. Park and M. E. J. Newman, Phys Rev E 70, 066117 (2004).

[30] D. Garlaschelli and M. I. Loffredo, Phys. Rev. E 78, 015101 (2008).

[31] K. Anand and G. Bianconi, Phys Rev E 80, 045102(R) (2009).

[32] M. Boguñá and R. Pastor-Satorras, Phys Rev E 68, 036112 (2003).

[33] P. Białas, Z. Burda, and B. Wacław, AIP Conf Proc 776, 14 (2005).

[34] N. Berger, C. Borgs, J. T. Chayes, and A. Saberi, Ann Probab 42, 1 (2014).

[35] P. W. Holland and S. Leinhardt, J. Am. Stat. Assoc. 76, 33 (1981). 
[36] O. Frank and D. Strauss, J Am Stat Assoc 81, 832 (1986).

[37] G. Caldarelli, A. Capocci, P. D. L. Rios, , and M. A. M. noz, Phys Rev Lett 89, 258702 (2002).

[38] S. Wasserman and P. E. Pattison, Psychometrika 61, 401 (1996).

[39] C. J. Anderson, S. Wasserman, and B. Crouch, Social Networks 21, 37 (1999).

[40] G. Robins, P. Pattison, Y. Kalish, and D. Lusher, Social Networks 29, 173 (2007).

[41] K. Zuev, O. Eisenberg, and D. Krioukov, J. Phys. A: Math. Theor. 48, 465002 (2015).

[42] F. Papadopoulos, M. Kitsak, M. Serrano, M. Boguñá, and D. Krioukov, Nature 489, 537 (2012).

[43] D. Krioukov, F. Papadopoulos, M. Kitsak, A. Vahdat, and M. Boguñá, Phys. Rev. E 82, 036106 (2010).

[44] T. Squartini and D. Garlaschelli, New Journal of Physics
13, 083001 (2011).

[45] P. Colomer-de Simon and M. Boguñá, Phys. Rev. E 86, 026120 (2012).

[46] R. Albert and A.-L. Barabási, Rev Mod Phys 74, 47 (2002).

[47] D. Krioukov, M. Kitsak, R. S. Sinkovits, D. Rideout, D. Meyer, and M. Boguñá, Sci Rep 2, 793 (2012).

[48] R. Aldecoa, C. Orsini, and D. Krioukov, Comput Phys Commun 196, 492 (2015).

[49] V. I. Arnold, Mathematical Methods of Classical Mechanics (Springer, New York, 2010).

[50] K. Anand, D. Krioukov, and G. Bianconi, Phys Rev E 89, 062807 (2014).

[51] T. Squartini, J. D. Mol, F. D. Hollander, and D. Garlaschelli, Phys. Rev. Lett. 115, 268701 (2015). 